\title{
Pivampicillin compared with erythromycin for treating women with genital Chlamydia trachomatis infection
}

\author{
MARIE CRAMERS, * PIA KASPERSEN, $\dagger$ ELliS FROM,* BIRGER R MøLLER $\dagger$ \\ From the Departments of *Dermatology and Venereology, †Obstetrics and Gynaecology, University Hospital, \\ Aarhus, Denmark
}

SUMMARY In a randomised single blind study, pivampicillin was compared with erythromycin in women with urogenital Chlamydia trachomatis infections. The pivampicillin dosage was $700 \mathrm{mg}$ twice a day and the erythromycin dosage $500 \mathrm{mg}$ twice a day for seven days. Follow up took place on days 7 and 14 after the start of treatment.

All 26 women treated with pivampicillin were culture negative for chlamydiae at the first and second follow up visits. All 23 women who received erythromycin were culture negative at the first follow up visit, but one was culture positive at the second follow up visit.

Gastrointestinal side effects were recorded in five patients receiving pivampicillin and in nine receiving erythromycin. Two patients receiving erythromycin were withdrawn from treatment because of gastrointestinal disturbances, compared with none receiving pivampicillin.

Chlamydia trachomatis is probably the most common sexually transmitted organism in the Western World, and it causes cervicitis, urethritis, endometritis, endosalpingitis, periappendicitis, and perihepatitis in women. ${ }^{\prime}$ Tetracyclines or erythromycin are recommended to treat genital chlamydial infections, whereas penicillins have been regarded as ineffective. ${ }^{2}$

In a preliminary publication we reported excellent results of 10 day treatment with pivampicillin in eradicating $C$ trachomatis from the genital tracts of women. ${ }^{3}$ That study, however, did not evaluate the optimum dose and did not compare the efficacy of treatment with that of generally accepted first line drugs. In the study reported here we therefore compared the effect of pivampicillin with that of erythromycin in treating genital chlamydial infection in women.

\section{Patients and methods}

PATIENTS

We included in a randomised single blind (investigator blinded) study 49 women from whose cervices or

Address for reprints: Dr Birger Møller, Department of Obstetrics and Gynaecology, Rigshospitalet, DK-2100 Copenhagen, Denmark

Accepted for publication 23 December 1987 urethras, or both, $C$ trachomatis had been cultured, but who were culture negative for Neisseria gonorrhoeae.

\section{STUDY DESIGN}

At the first visit of each patient to the department of venereology of this hospital, urethral and cervical material was taken for culture for $C$ trachomatis and $N$ gonorrhoea. We investigated patients who were culture positive for chlamydiae but negative for gonococci. Each patient received either pivampicillin $700 \mathrm{mg}$ twice a day for seven days or erythromycin stearate $500 \mathrm{mg}$ twice a day for seven days, to be taken immediately before meals. We asked patients to abstain from sexual intercourse during the investigation. Urethral and cervical specimens were cultured for $C$ trachomatis at follow up on days 7 and 14 after the start of treatment.

We excluded patients who had taken antibiotics in the preceding month or who were allergic to penicillin or erythromycin. We traced and treated all sexual contacts.

\section{Results}

Pivampicillin was given to 26 women (mean age 21 (range 18 to 27) ), and erythromycin to 23 women (mean age 21 (range 18 to 26) ). No bacteriological 
treatment failures were found in patients treated with pivampicillin (all patients were culture negative for $C$ trachomatis at the first and second follow up visits, except two who did not attend for the second follow up). All 23 treated with erythromycin were culture negative for chlamydiae at the first follow up, but one woman was culture positive at the second follow up. She denied the possibility of reinfection.

Gastrointestinal side effects were recorded in five patients receiving pivampicillin and in nine receiving erythromycin. Two receiving erythromycin were withdrawn after a few days of treatment because of gastrointestinal disturbances, whereas all patients receiving pivampicillin completed the treatment. One patient treated with pivampicillin developed a maculopapular eruption of the skin three to four days after the end of treatment; no skin eruptions were observed in those receiving erythromycin.

\section{Discussion}

Antibiotics may be classified as being in one of three groups according to their in vitro activity against $C$ trachomatis: those with high activity (minimum inhibitory concentrations (MIC) less than $0.06 \mathrm{mg} / \mathrm{l}$ ) include tetracycline and erythromycin, those with intermediate activity (MIC $0 \cdot 25-4.0 \mathrm{mg} / \mathrm{l}$ ) include pivampicillin, and those with low activity (MIC 64$256 \mathrm{mg} / \mathrm{l}$ ) include trimethoprim and metronidazole.

The study design included a second follow up examination seven days after the end of treatment. A longer period of surveillance would probably have shown more treatment failures. The increased risk of reinfection during a longer follow up period, however, makes it difficult to distinguish whether a positive chlamydial culture is a result of reinfection or of treatment failure.

Erythromycin is a well established drug in treating urogenital chlamydial infection. In different studies, doses of $500 \mathrm{mg}$ twice daily for 10 to 14 days resulted in incidences of reisolation of $0 \%$ to $22 \%{ }^{4}$

Amoxycillin $1.5 \mathrm{~g}$ a day for 10 days given to nine pregnant women with chlamydial infection resulted in reisolation from three women after delivery.' Alexander and Harrison, however, reported that seven day treatment with $1.5 \mathrm{~g}$ amoxycillin was effective in eradicating cervical chlamydial infection. ${ }^{6}$

Pivampicillin as an alternative to first line drugs (erythromycin and tetracyclines) for treating chlamydial infections was studied by Johannisson $e t$ $a l$, who found that five of 22 men with urogenital chlamydial infections were culture negative after treatment with pivampicillin for seven days, whereas eight complained of persistent symptoms. ${ }^{7}$ Similarly, Hagdrup et al found that only seven of 14 chlamydia positive patients were cured after treatment with $500 \mathrm{mg}$ pivampicillin three times a day for seven days. ${ }^{8}$ In contrast to those results, we found in a previous study $^{3}$ as well as in our present investigation that pivampicillin is an acceptable alternative to erythromycin for treating uncomplicated lower genital tract infection caused by $C$ trachomatis.

\section{References}

1 Mårdh P-A. Ascending chlamydial infection in the female genital tract. In: Oriel D, Ridgway G, Schachter J, Taylor-Robinson D, Ward M, eds. Chlamydial infections. Cambridge: Cambridge University Press, 1986:173-84.

2 Bowie WR. Etiology and therapy of Chlamydia trachomatis infections. Drugs 1984;27:459-68.

3 Möller BR, Cramers M, From E. Pivampicillin in treating genital infection with Chlamydia trachomatis. Genitourin Med 1985;61:264-5.

4 Oriel JD. Chemotherapy. In: Oriel D, Ridgway G, Schachter J, Taylor-Robinson D, Ward M, eds. Chlamydial infections. Cambridge: Cambridge University Press, 1986:513-23.

5 Bell TA, Sandstrom IK, Eschenbach DA, et al. Treatment of Chlamydia trachomatis in pregnancy with amoxicillin. In: Mårdh P-A, Holmes KK, Oriel JD, Piot P, Schachter J, eds Chlamydial infections Amsterdam: Elsevier Biomedical Press, 1982:221-4.

6 Alexander ER, Harrison HR. Role of Chlamydia trachomatis in perinatal infection. Rev Infect Dis 1984;5:713-9.

7 Johannisson G, Sernryd A, Lycke E. Susceptibility of Chlamydia trachomatis to antibiotics in vitro and in vivo. Sex Transm Dis 1979;6:50-7.

8 Hagdrup H, Kristensen J, Scheibel J. Failure of pivampicillin in treating chlamydial infections. British Journal of Venereal Diseases 1984;60:204. 